\title{
Solvent effects on intermolecular electron transfer processes
}

\author{
Carlos M. Previtali \\ Department of Chemistry and Physics \\ Universidad Nacional de Río Cuarto, 5800 Río Cuarto, Argentina
}

\begin{abstract}
The solvent effects on the rate constants and activation parameters of several intermolecular electron transfer reactions were investigated. The studies were carried out in protic (methanol, ethanol) and aprotic (acetonitrile, propionitrile) solvents. It was found that rate constants are generally higher in the protic solvents and that activation enthalpies are generally small and in some cases negative. The negative values were found in acetonitrile, while for the same reaction positive values are observed in alcoholic solvents. Large negative values of activation entropies were observed. They are more negative in the aprotic solvents. These observations can be explained by considering the entropy changes caused by the solvent reorganization on forming the transition state. The solvent effect on the quantum yield of the charge separation process was also investigated. For the system pyrene-indole the free-ions quantum yield changes in accordance with the trend of the cage escape rate constant. On the other hand, in the triplet quenching of dibenz-anthracene by nitrobenzenes the charge separation process is much less efficient in acetonitrile than in methanol. These results cannot be explained if the solvent is considered as a dielectric continuum.
\end{abstract}

\section{INTRODUCTION}

In spite of the large amount of work on photoinduced electron transfer reactions the solvent effect on the rate constants and activation parameters for intermolecular processes have received little consideration until recently [1]. There remain several aspects of these effects that are not satisfactorily explained by the simple Rehm-Weller-Marcus model.

During the last years we have been involved in the study of the solvent effects on the rate constants and activation parameters of several intermolecular electron transfer reactions. The reactions investigated were the luminescence quenching of $\mathrm{Ru}(\mathrm{bpy}){ }_{3}{ }^{+2}$ by aromatic amines [2] and electron acceptors [3], the quenching of the triplet sate of polycyclic aromatic hydrocarbons (PAHs) by nitrobenzenes [4], the electron transfer reaction between excited singlet pyrene and indole, and the electron transfer from excited singlet PAHs to electron acceptors. The solvent effect on the charge separation efficiencies was also investigated in some of these systems. These studies were carried out in polar solvents of similar macroscopic dielectric properties with the goal of observing specific effects of the solvent structure on the kinetics of these reactions. In this sense protic (methanol, ethanol) and aprotic (acetonitrile $(\mathrm{MeCN})$, propionitrile $(\mathrm{PrCN})$ ) solvents were employed.

\section{SPECIFIC SOLVENT EFFECTS ON THE RATE CONSTANT}

The most remarkable finding was that in two solvents of similar macroscopic dielectric properties such as methanol $\left(\varepsilon=32.6, \mathrm{n}_{D}=1.331\right)$ and acetonitrile $\left(\varepsilon=35.9, \mathrm{n}_{\mathrm{D}}=1.342\right)$ the rate constants may differ in some cases by more than one order of magnitude. An example of such effect can be observed in Table 1 for the case of the excited state quenching of $\mathrm{Ru}(\mathrm{bpy}) 3^{+2}$ by aromatic amines and nitrobenzenes. In all cases the rate constants are higher in $\mathrm{MeOH}$ than in $\mathrm{MeCN}$. Higher rate constants in $\mathrm{MeOH}$ have also been observed in the electron transfer quenching of the triplet state of PAHs by nitrobenzenes. Some examples are shown in Table 2. Also in the electron transfer quenching of excited singlet states of PAHs by nitro and cyanobenzenes, the rate constants happen to be generally higher in methanol than in acetonitrile. Results pertaining to this latter system are shown in Table 3. 
TABLE 1. Electron transfer quenching of excited

$\mathrm{Ru}(\mathrm{bpy})_{3}{ }^{+2}$ by organic electron donors and acceptors. Bimolecular rate constants at $298 \mathrm{~K}$.

\begin{tabular}{|l|c|c|}
\hline & \multicolumn{2}{|c|}{$\mathrm{k}_{\mathrm{q}} / \mathrm{M}^{-1} \mathrm{~s}^{-1}$} \\
\hline \multicolumn{1}{|c|}{ Quencher } & Acetonitrile & Methanol \\
\hline 1 - Naphthylamine & $8 \times 10^{8}$ & $1.9 \times 10^{9}$ \\
2 - Naphthylamine & $2.3 \times 10^{7}$ & $3.4 \times 10^{8}$ \\
N,N'- Dimethylaniline & $9.5 \times 10^{7}$ & $1.6 \times 10^{8}$ \\
$1,3-$ Dinitrobenzene & $1.9 \times 10^{9}$ & $2.3 \times 10^{9}$ \\
m-Nitrobenzaldehyde & $5.0 \times 10^{7}$ & $1.4 \times 10^{8}$ \\
p-Chloronitrobenzene & $8.8 \times 10^{6}$ & $1.3 \times 10^{8}$ \\
\hline
\end{tabular}

These results are not easily explained by the classical Marcus electron transfer theory. In this model the rate constant for a bimolecular electron transfer proceeding by the following simple mechanism:

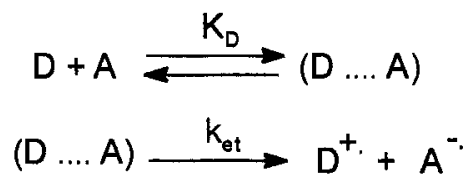

is given by [5]

$$
k_{e t}=K_{D} \kappa_{e t} v_{n} \exp \left(-\frac{\left(\lambda+\Delta G^{o}\right)^{2}}{2 \lambda R T}\right)
$$

where $K_{D}$ is the equilibrium constant for the formation of the encounter complex, $v_{n}$ is a nuclear frequency of passage through the transition state and $\kappa_{e l}$ is the electronic transmission coefficient. In the classical treatment $\kappa_{e l}$ is usually taken as unity. $\Delta G^{o}$ is the Gibbs energy of the electron transfer reaction when the donor and acceptor are at a distance $r_{A D}$.

The reorganization energy $\lambda$ has two contributions:

$$
\lambda=\lambda_{\text {in }}+\lambda_{\text {out }}
$$

The inner term $\lambda_{i n}$ arises from structural changes on going from the equilibrium configuration of the reactants to that of the products. It is a function of bond lengths and force constants in both states. The outer term $\lambda_{\text {out }}$ is called the solvent reorganization energy and it is often the major contribution to the total reorganization energy. When a dielectric continuum model is applied the solvent contribution can be written as [5]

$$
\lambda_{o u t}=B\left[\frac{1}{\varepsilon_{o p}}-\frac{1}{\varepsilon_{s}}\right]=B \gamma
$$


where $\varepsilon_{\mathrm{op}}$ and $\varepsilon_{\mathrm{s}}$ are the optical and static dielectric constants respectively, and $B$ is a function of the model and molecular dimensions that is usually taken as solvent independent.

TABLE 2: Rate constants at $298 \mathrm{~K}$ for the bimolecular quenching of triplet state of polycyclic hydrocarbons by nitrobenzenes

\begin{tabular}{|c|c|c|c|}
\hline & & \multicolumn{2}{|c|}{$\mathrm{k}_{\mathrm{q}} / \mathrm{M}^{-1} \mathrm{~s}^{-1}$} \\
\hline PAH & Quencher & Acetonitrile & Methanol \\
\hline Anthracene & p-nitrobenzaldehyde & $2.4 \times 10^{7}$ & $3.1 \times 10^{7}$ \\
\hline Anthracene & 1,3-Dinitrobenzene & $3.6 \times 10^{7}$ & $2.1 \times 10^{8}$ \\
\hline a,h-Dibenzanthracene & m-Nitrobenzaldehyde & $1.2 \times 10^{9}$ & $1.2 \times 10^{9}$ \\
\hline a,h-Dibenzanthracene & p-Chloronitrobenzene & $4.8 \times 10^{8}$ & $1.3 \times 10^{9}$ \\
\hline
\end{tabular}

TABLE 3. Rate constants for the electron transfer singlet quenching of polycylic aromatic hydrocarbons by electron acceptors

\begin{tabular}{|c|c|c|c|}
\hline & & \multicolumn{2}{|c|}{$\mathrm{k}_{\mathrm{q}} / \mathrm{M}^{-1} \mathrm{~s}^{-1}$} \\
\hline PAH & Quencher & Acetonitrile & Methanol \\
\hline $\begin{array}{l}\text { a,h-Dibenzanthracene } \\
\text { Pyrene } \\
\text { a,h-Dibenzanthracene } \\
\text { Pyrene } \\
\text { Naphthalene } \\
\text { a,h-Dibenzanthracene } \\
\text { a,h-Dibenzanthracene } \\
\text { Pyrene }\end{array}$ & $\begin{array}{l}\text { 1,2-dicyanobenzene } \\
\text { 3-cyanopyridine } \\
\text { 1,3-dicyanobenzene } \\
\text { 4-chlorobenzonitrile } \\
\text { benzonitrile. } \\
\text { 3-cyanopyridine } \\
\text { 4-chlorobenzonitrile } \\
\text { benzonitrile. }\end{array}$ & $\begin{array}{l}3.1 \times 10^{9} \\
2.9 \times 10^{8} \\
1.1 \times 10^{8} \\
1.0 \times 10^{8} \\
6.5 \times 10^{6} \\
1.0 \times 10^{6} \\
9.9 \times 10^{6} \\
9.1 \times 10^{5}\end{array}$ & $\begin{array}{l}7.9 \times 10^{9} \\
3.9 \times 10^{9} \\
1.9 \times 10^{8} \\
8.1 \times 10^{7} \\
1.6 \times 10^{7} \\
6.0 \times 10^{7} \\
4.3 \times 10^{7} \\
1.2 \times 10^{6}\end{array}$ \\
\hline
\end{tabular}

According to Marcus classical theory the solvent may influence the electron transfer rate constant by altering several of the parameters involved. First, the driving force $\Delta G^{\circ}$ can be altered by different stabilization of the product ion pair. Second the solvent contribution to the reorganization energy $\lambda_{\text {out }}$ is modified through changes in the static and optical dielectric constants. Finally, the nuclear frequency may be expected to be solvent dependent if it is predominantly a solvent mode [6]. For the solvents under consideration, $\gamma$ in eq (4) is practically the same $(0.523$ for $\mathrm{MeCN}, 0.501$ for $\mathrm{PrCN}, 0.536$ for $\mathrm{MeOH}$ and 0.499 for $\mathrm{EtOH}$ at room temperature) and the driving force for a given reaction is not expected to differ significantly from one solvent to another, since the dielectric constants are very similar. Therefore the observed solvent effects on the rate constants are can not be explained by changes in the exponential term if a continuum model of the solvent is considered. In the preexponential term $\mathrm{K}_{\mathrm{D}}$ is at a first approximation solvent independent, and consequently the nuclear frequency might be the main factor responsible for the changes in the rate constant. 


\section{SOLVENT EFFECTS ON THE ACTIVATION PARAMETERS}

In order to get a better understanding of this solvent effect, the temperature dependence of the rate constants was investigated and the activation parameters were determined. In terms of the Transition State Theory the rate constant was expressed as:

$$
k_{e t}=\frac{k T}{h} \exp \left(\frac{\Delta S^{\ddagger}}{R}\right) \exp \left(-\frac{\Delta H^{\ddagger}}{R T}\right)
$$

This can be related to eq (1) by noticing that the nuclear frequency has been replaced by the universal frequency term $k T / h$. Therefore all changes in the nuclear frequency are now included in the activation entropy.

Activation enthalpies and entropies were determined by plots of $\ln \left(k_{e f} / T\right)$ vs. $1 / T$. In Tables 4 and 5 the activation parameters for the rate constants of Tables 1 and 2 are collected. Other system for which the solvent effect on rate constants and activation parameters was investigated, is the electron transfer quenching of the first excited singlet of pyrene by indole. The results for this reaction are presented in Table 6. In general large negative values of activation entropies are observed. It emerges from Tables 4 to 6 that for all the reactions the activation entropy changes are more negative in the aprotic solvents.

TABLE 4. Activation Parameters for the electron transfer quenching of excited $\mathrm{Ru}(\mathrm{bpy}) 3^{+2}$ by organic acceptors and donors.

\begin{tabular}{|l|c|c|c|c|}
\hline \multicolumn{1}{|c|}{ Solvent } & \multicolumn{2}{|c|}{ Acetonitrile } & \multicolumn{2}{c|}{ Methanol } \\
\hline Quencher & $\begin{array}{c}\Delta \mathrm{H}^{\ddagger} \\
(\mathrm{kcal} / \mathrm{mol})\end{array}$ & $\begin{array}{c}\Delta \mathrm{S}^{\ddagger} \\
(\mathrm{cal} / \mathrm{K} \mathrm{mol})\end{array}$ & $\begin{array}{c}\Delta \mathrm{H}^{\ddagger} \\
(\mathrm{kcal} / \mathrm{mol})\end{array}$ & $\begin{array}{c}\Delta \mathrm{S}^{\ddagger} \\
(\mathrm{cal} / \mathrm{K} \mathrm{mol})\end{array}$ \\
\hline 1-Naphthylamine & 2.2 & -13.5 & 2.1 & -9.0 \\
2-Naphthylamine & 3.1 & -14.2 & 3.0 & -9.4 \\
N,N'-Dimethylaniline & 2.2 & -14.5 & 3.2 & -10.0 \\
1,3-Dinitrobenzene & -2.5 & -21.0 & 0.5 & -13.0 \\
m-nitrobenzaldehyde & -3.5 & -35.0 & 2.2 & -17.0 \\
p-Chloronitrobenzene & -2.6 & -35.0 & 0.2 & -21.0 \\
\hline
\end{tabular}

These observations can be explained by considering the entropy changes caused by the solvent reorganization on forming the transition state. In all the reactions under consideration one of the reactants is neutral. When the electron transfer takes place a new charge asymmetry develops. The polar solvent molecules must adopt specific orientations for charge stabilization. This may be considered as a "freezing" of the solvent molecules in the transition state and results in a negative activation entropy. In a solvent like methanol, the molecules are in a more ordered structure in the initial state due to hydrogen bonding, therefore the entropy change for reaching the transition state will be less than in an aprotic solvent like acetonitrile.

The activation entropy changes may also be related to the nuclear frequency in eq (1). It is hard to make an estimation of $v_{n}$ and how it is going to be affected by the solvent properties. In the classical model $v_{n}$ can be written as [7]

$$
v_{n}=\left(\frac{v_{\text {out }}^{2} \lambda_{\text {out }}+v_{\text {in }}^{2} \lambda_{\text {in }}}{\lambda}\right)^{1 / 2}
$$


If the internal reorganization energy is very much lower than the solvent reorganization, as is normally the case in electron transfer processes involving organic molecules, then the major contribution to $v_{n}$ comes from the low frequency solvent modes, which are normally in the order of $10^{12} \mathrm{~s}^{-1}$. The preexponential factor $\mathrm{K}_{\mathrm{D}} v_{n}$ was estimated as $3.5 \times 10^{11} \mathrm{M}^{-1} \mathrm{~s}^{-1}$ in $\mathrm{MeCN}$ and $1 \times 10^{12} \mathrm{M}^{-1} \mathrm{~s}^{-1}$ in $\mathrm{MeOH}$ for the triplet quenching of PAHs by nitrobenzenes [4]. Assuming a similar $K_{D}$ a higher frequency results for $\mathrm{MeOH}$ which may arise from the stronger intermolecular interaction in this solvent.

In Tables 4 to 6 it can be seen that for a given reaction the activation enthalpy is smaller in the aprotic solvents. In many cases negative activation enthalpies are observed in acetonitrile or propionitrile. Only for the quenching of pyrene excited singlet by indole a slightly negative activation enthalpy occurs also in methanol.

TABLE 5. Activation Parameters for the triplet quenching of polycyclic aromatic hydrocarbons by nitrobenzenes in $\mathrm{MeOH}$ and $\mathrm{MeCN}$

\begin{tabular}{|l|l|c|c|c|c|}
\hline & Solvent & \multicolumn{2}{|r|}{ Acetonitrile } & \multicolumn{2}{c|}{ Methanol } \\
\hline \multicolumn{1}{|c|}{ PAH } & Quencher & $\begin{array}{c}\Delta \mathrm{H}_{\ddagger}^{\ddagger} \\
(\mathrm{kcal} / \mathrm{mol})\end{array}$ & $\begin{array}{c}\Delta \mathrm{S}^{+} \\
(\mathrm{cal} / \\
\mathrm{K} \mathrm{mol})\end{array}$ & $\begin{array}{c}\Delta \mathrm{H}^{\ddagger} \\
(\mathrm{kcal} / \mathrm{mol})\end{array}$ & $\begin{array}{c}\Delta \mathrm{S}^{\ddagger} \\
(\mathrm{cal} / \\
\mathrm{K} \mathrm{mol})\end{array}$ \\
\hline Anthracene & p-nitrobenzaldehyde & +0.8 & -22.0 & +4.0 & -11.0 \\
Anthracene & 1,3 -Dinitrobenzene & +0.5 & -23.0 & +2.4 & -12.0 \\
a,h-Dibenzanthracene & m-Nitrobenzaldehyde & -1.6 & -23.0 & +3.0 & -7.0 \\
a,h-Dibenzanthracene & p-Chloronitrobenzene & -1.4 & -24.0 & +0.8 & -14.0 \\
\hline
\end{tabular}

TABLE 6. Rate constants and activation parameters for the electron transfer quenching of pyrene excited singlet by indole.

\begin{tabular}{|c|c|c|c|}
\hline solvent & $\mathrm{k}_{\mathrm{q}} / \mathrm{M}^{-1} \mathrm{~s}^{-1}$ & $\begin{array}{c}\Delta \mathrm{H}^{\ddagger} \\
(\mathrm{kcal} \mathrm{mol}-1)\end{array}$ & $\begin{array}{c}\Delta \mathrm{S} \ddagger \\
(\mathrm{cal} / \mathrm{K} \mathrm{mol})\end{array}$ \\
\hline Acetonitrile & $2.4 \times 10^{8}$ & -2.9 & -30.0 \\
Propionitrile & $1.1 \times 10^{8}$ & -1.4 & -26.4 \\
Methanol & $1.5 \times 10^{8}$ & -0.2 & -21.8 \\
Ethanol & $6.4 \times 10^{7}$ & 0.0 & -22.8 \\
\hline
\end{tabular}

Negative activation energies in electron transfer processes have been previously observed in thermal reactions involving metallic complexes [8], in the triplet quenching of PAHs in acetonitrile [9] and for the excited singlet quenching of aromatic molecules [1]. Since this effect appears in reacting systems of such diversity it must be concluded that it is a general feature of electron transfer reactions. Two mechanisms have been proposed for explaining it. The first [1,9] appeals to the formation of an exciplex, which in some cases can be strongly bound, such that its formation enthalpy exceeds the activation energy of the electron transfer step. The second one, which we consider the most likely, was proposed by Marcus and Sutin [8]. It is based on the large negative values observed for the standard entropy changes in electron transfer reactions. This implies a lower density of quantum states in the product's side of the potential energy surface. Then, by a simple application of Tolman's theorem, the observation of negative activation energies can be explained [8]. Since very large negative activation entropies are observed in the aprotic solvents, it is 
most likely that the overall entropy change is also large and negative, and it is precisely in these cases where negative values for the activation enthalpies are observed.

\section{SOLVENT EFFECTS ON THE CHARGE SEPARATION EFFICIENCY}

The solvent effect on the quantum yield of the charge separation process was also investigated by laser flash photolysis. The transient absorption spectra of the long lived species formed in the quenching process were determined and the formation quantum yields were measured in order to prove the electron transfer of the reaction in all the solvents, and to determined the fate of the radical ion pair initially formed. In the case of the quenching of a, h-dibenzanthracene (DBA) triplets by nitrobenzenes it was possible to demonstrated that the only process for the geminate ion pair decay was the separation in free radical ions or the decay to the ground state. Transient absorption spectrum for these systems can be seen in Fig. 1.

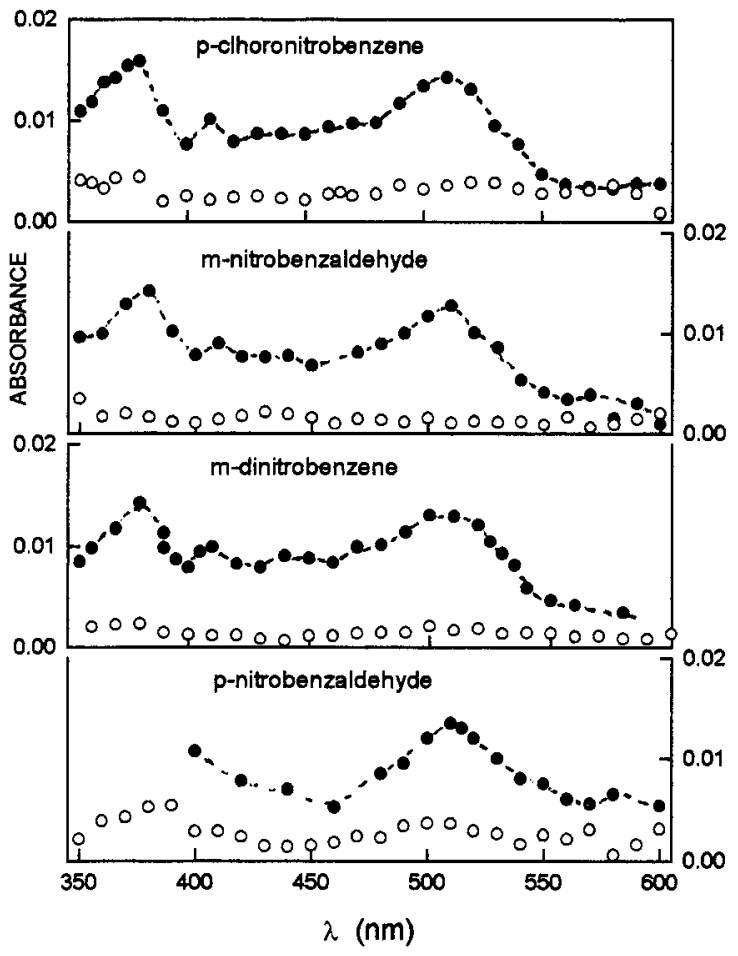

Fig. 1 Transient absorption spectra taken $20 \mu \mathrm{s}$ after the laser pulse for $D B A$ in the presence of nitrobenzenes in $\mathrm{MeOH}$ ( $\bullet$ ) and $\mathrm{MeCN}$ (o).
The bands centered around 380 and 510 $\mathrm{nm}$ can be ascribed to the DBA radical cation. The transient spectra were taken under conditions of equal absorption of the laser pulse by DBA ground state and the same fraction of triplet intercepted by the quencher in all cases (90\%). The free radical ion quantum yields are collected in Table 7. While in methanol the radical ion is formed in high yield in acetonitrile it is almost absent This is a totally unexpected result, specially for a process originated in the triplet state. Moreover, in the aprotic solvent, being a less viscous one, the cage escape is expected to be higher than in methanol and consequently a higher charge separation efficiency should be apparent. This results cannot be explained if the solvent is considered as a dielectric continuum. The only explanation for this solvent effect is the operation of a strong interaction of the quencher molecules that alter the nature or the kinetic behavior of the geminate ion pair.

TABLE 7. Radical ion quantum yields during the quenching of DBA triplet state by nitrobenzenes

\begin{tabular}{|c|c|c|}
\hline Quencher & $\mathrm{MeOH}$ & $\mathrm{MeCN}$ \\
\hline p-Nitobenzaldehyde & $0.6 \pm 0.1$ & 0.1 \\
p-Clhoronitrobenzene & $0.6 \pm 0.1$ & 0.1 \\
m-Nitrobenzene & $0.5 \pm 0.1$ & $<0.05$ \\
m-Dinitrobenzene & $0.5 \pm 0.1$ & $<0.05$ \\
\hline
\end{tabular}

The solvent effect on the charge separation process was also studied in the reductive quenching of singlet excited pyrene by indole. The absorption spectrum of the long lived species after the quenching is shown in Fig. 2 for four solvents. The spectra were taken, also in this case, under conditions of equal absorption of the laser pulse and the same fraction of singlet quenching.

In the absence of indole the typical triplet absorption of pyrene is observed. In the presence of indole at a concentration such that near $90 \%$ of the excited singlets are intercepted, two species are predominant in the spectrum in all cases, the triplet state $\left(\lambda_{\max }=414 \mathrm{~nm}[10]\right)$ and the pyrene radical anion $\left(\lambda_{\max }=490 \mathrm{~nm}[11]\right)$. The triplet yields are very much higher than those expected according to the fraction of excited singlet states quenched. Consequently it can be concluded that the quenching process 
leads in part to the triplet sate. The radical ion yield presents large differences from one solvent to other. However in this system this yield changes in accordance with the trend in the cage escape rate constant.
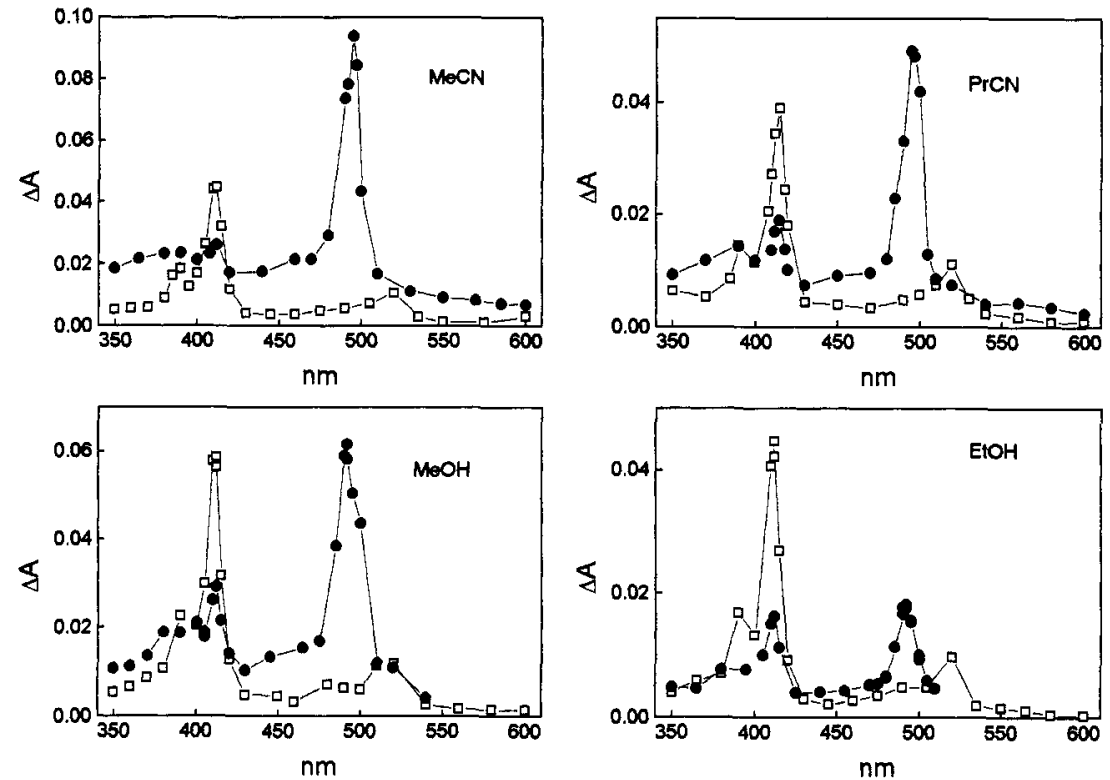

Fig. 2: Transient absorption spectra of pyrene in the absence $(\square)$ and the presence of indole $(\bullet)$ taken immediately after the laser pulse.

These results may be explained by the following simple reaction scheme:

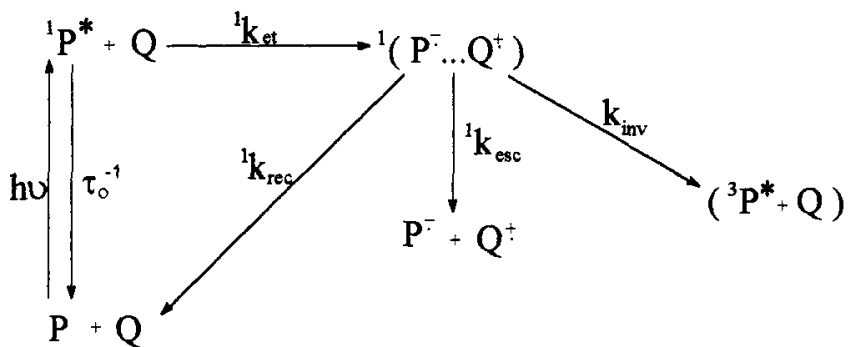

In the time scale of our laser photolysis experiments, the pyrene radical anions observed are those that escape the cage recombination processes. According to the scheme the free ions quantum yield can be written as:

$\Phi_{i o n}=f \times \frac{{ }^{1} k_{e s c}}{{ }^{1} k_{e s c}+k_{i n v}+k_{r e c}}$

where $f$ is the fraction of singlets

quenched. Equation (7) can be re-written as:

$$
\frac{\Phi_{\text {ion }}}{f-\Phi_{\text {lon }}}=\frac{{ }^{1} k_{\text {esc }}}{{ }^{1} k_{\text {rec }}+k_{\text {inv }}}
$$

Hence a plot of the left hand side vs. ${ }^{1} k_{\text {esc }}$ should result in a straight line going through the origin. In order to estimate $k_{\text {esc }}$ we made use of the Eigen equation [12]:

$$
{ }^{1} k_{e s c}=\left(\frac{2 k_{B} T}{\pi \eta r^{2}}\right)\left(\frac{z_{A} z_{B} e^{2}}{\varepsilon r k_{B} T}\right)\left[1-\exp \left(-\frac{z_{A} z_{B} e^{2}}{\varepsilon r k_{B} T}\right)\right]^{-1}
$$

where $\eta$ and $\varepsilon$ are the solvent viscosity and dielectric constant, respectively. $z_{A}$ and $z_{B}$ are the charges of the separating ions initially at a distance $r$. Assuming $0.7 \mathrm{~nm}$ for $r$ eq (9) affords $6 \times 10^{9} \mathrm{sec}^{-1}$ for ${ }^{1} k_{\text {esc }}$ in $\mathrm{MeCN}$. This was re-scaled by a factor $1 / 6$ in order to agree with the experimental results in MeCN [13].

A plot of the results of radical ion quantum yield according to eq (8) for the system pyrene-indole can be seen in Fig. 3. 


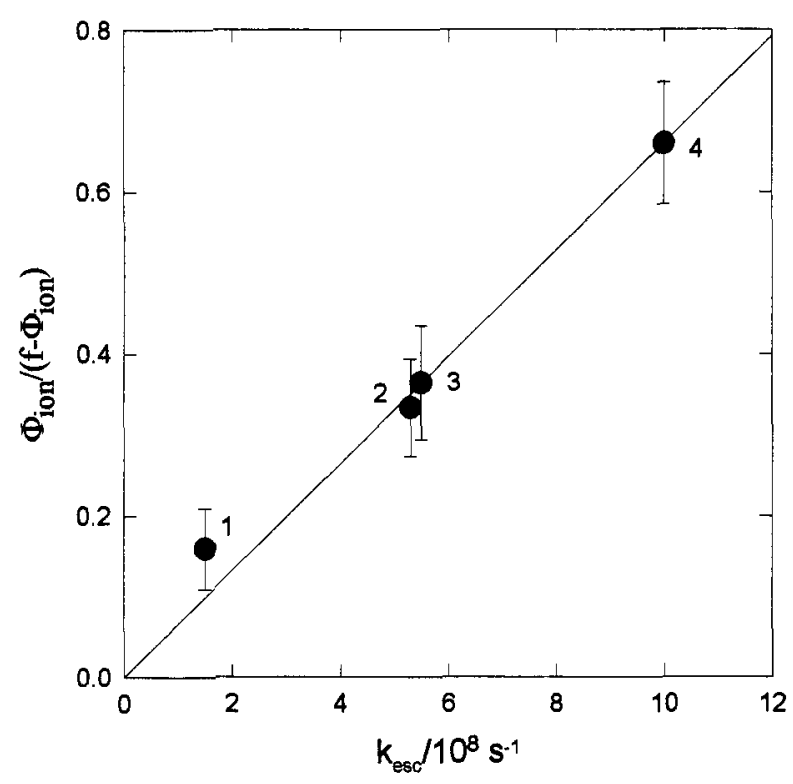

Figure 3. Plot of the left hand side of eq (8) vs. $k_{\text {esc. }}$. Solvent key: $I=E t O H, 2=M e O H$, $3=\operatorname{PrCN}, 4=\mathrm{MeCN}$.

Within the experimental error of the data, a linear plot with zero intercept is obtained. It can be concluded that for this system the macroscopic solvent properties give a good account of the observed behavior. Moreover, according to eq (8) the linearity of the plot implies a constant value of ${ }^{1} k_{\text {rec }}+k_{i m v}$, e.g., the geminate ion-pairs formed in the excited singlet quenching of pyrene by indole decay to ground state or triplet state with a solvent independent total rate constant. This result is most remarkable since it is independent of the protic or aprotic nature of the solvent.

In summary, specific solvent effects are observed in bimolecular electron transfer reactions that can not be explained by considering the solvent as a dielectric continuum. A microscopic model that contemplates the molecular structure and its interaction with the reacting molecules of the solvent is necessary.

ACKNOWLEDGMENTS: Thanks are given to G. Zanini and H. Montejano who collaborated in the unpublished work here presented. The financial support from Consejo Nacional de Investigaciones Científicas y Técnicas (CONICET), Consejo de Investigaciones Científicas y Tecnológicas de la Provincia de Córdoba (CONICOR) and Secretaría de Ciencia y Técnica de la Universidad Nacional de Río Cuarto, is gratefully acknowledged.

\section{REFERENCES}

[1] For a review of some earlier work see J. E. Baggott in "Photoinduced Electron Transfer" (Eds. M.A. Fox and M. Chanon) Part B, p.385, Elsevier, New York, 1988.

[2] H.A. Garrera, J.J. Cosa and C.M. Previtali, J. Photochem. Photobiol. A, Chem., 47, 147 (1989).

[3] H.A. Garrera, J.J. Cosa and C.M. Previtali, J. Photochem. Photobiol. A, Chem., 56, 267 (1991).

[4] H.A. Montejano, V. Avila, H.A. Garrera and C.M. Previtali, J. Photochem. Photobiol. A, Chem., 72, 117 (1993).

[5] R.A. Marcus and N. Sutin, Biochim. Biophys. Acta, 811, 265 (1985).

[6] N. Sutin, Acc. Chem. Res., 9, 275 (1982).

[7] M. Grätzel, Heterogeneous Photochemical Electron Transfer, p. 4, CRC Press, Boca Raton, 1989.

[8] R. A.Marcus and N. Sutin, Inorg. Chem. 14, 213 (1975).

[9] V.A. Kuzmin, I.V. Renge and Yu. E. Borisevich, Chem. Phys. Lett., 70, 257 (1980).

[10] I. Carmichael and G. L. Hug, J. Phys. Chem. Ref. Data, 15 (1986) 1.

[11] T. Okada, I. Karaki and N. Mataga, J. Am. Chem. Soc., 104 (1982) 7191.

[12] M. Eigen, Z. Phys. Chem. NF, I (1954) 176.

[13] N. Mataga, T. Asahi, Y. Kanda, T. Okada and T. Kakitani, Chem. Phys., 127 (1988) 249. 